\title{
Devices based on 2D materials for on-chip amplification of ionization charges
}

\section{A. Ciarrocchi*}

INFN-Pisa and University of Pisa, Department of Physics

E-mail: alberto.ciarrocchi@pi.infn.it

\section{F. Forti}

INFN-Pisa and University of Pisa, Department of Physics

E-mail: Francesco.Fortiepi.infn.it

\section{G. lannaccone}

University of Pisa, Department of Information Engineering

E-mail: giuseppe.iannaccone@unipi.it

\section{G. Fiori}

University of Pisa, Department of Information Engineering

E-mail: g.fiorieiet.unipi.it

\begin{abstract}
Pixels detectors are widely used ionizing radiation detection devices in high-energy physics (HEP) experiments. Segmented detectors have been employed for many years due to the need to simultaneously track the thousands of particles emerging from modern colliders. For more precise and accurate measurements one would like to have faster, less noisy and smaller pixels, but current technology imposes several limits on these characteristics. The aim of this work is to explore the possible applications of bi-dimensional materials such as Graphene or transition metal dichalcogenide monolayers (TMDs) to address these problems. In particular, one wants to determine whether nano-electronic devices based on 2D materials could be used to obtain built-in pre-amplification of the pixel signal, thus achieving better detection performance. The working principle is the field-effect modulation of the channel conductivity in a 2D material-based transistor, due to the presence of ionization charges in a silicon absorber. Several architectures are tested, and a final device of choice is presented, with a sketch of a realistic readout system and its noise figure. The conductance modulation due to incoming particles is found to be more than $30 \%$, resulting in a strong current signal, which leads to very favourable signal-to-noise ratios (SNR).
\end{abstract}

The 25th International workshop on vertex detectors

September 26-30, 2016

La Biodola, Isola d'Elba, ITALY

${ }^{*}$ Speaker. 


\section{Introduction}

Silicon pixel sensors are used in particle colliders to reconstruct the trajectories of charged particles in the proximity of the beam collision point. Several factors determine the effectiveness of a detector: time response, pixel size, noise, detector thickness, radiation hardness. Nowadays the hybrid pixel technology is the most common in HEP experiments, because it allows both high SNR and good radiation hardness [1]. However, these devices have limitations: the double-layered structure leads to an intrinsically higher material budget, and the need for bump bonding prevents from shrinking the pixel size. There is indeed a strong interest in developing (semi-)monolithic pixels, where the integration of sensor and electronics promises better performance, as well as thinner and more power-efficient pixels. A reduction in material budget would indeed minimise multiple scattering and energy loss, incresing the precision of vertex reconstruction and calorimetric measurements. On-detector signal amplification could be used to improve SNR and reduce the material budget $[2,3]$.

In this area the are currently two main lines of research [1,3]: one focused on integrating part or all of the electronics in a high-resistivity substrate (DEPFET, SOI), and another one concerned with the adaptation of CMOS substrates to particle detection (MAPS). While DEPFETs show high SNR and radiation hardness, their fabrication is very challenging and time-consuming, and the readout mechanisms are still quite slow. On the other side, MAPS benefit from a simpler and cheaper CMOS fabrication process, but the signal produced is weaker due to the small depletion region, and more subject to radiation degradation.

It would be very interesting to conceive a structure that has high SNR without being excessively complicate to fabricate. A possible way to achieve this could exploit the electronic properties of novel 2D materials, in order to achieve built-in signal pre-amplification. This works presents the results of numerical simulations of such devices.

\section{2D Material-based Radiation Detectors}

\subsection{Previous Works}

A great amount of research has been done on radiation detectors based on graphene and/or other 2D materials in the past years, due to intriguing electrical and optical properties [4]. These devices usually exploit the direct absorption of radiation by the material, and are sensitive to lowerenergy radiation (generally the IR-UV range). Also, this approach limits the detecting area to the actual 2D-material surface.

A few proposals for devices using 2D materials for ionizing radiation detection have been made, but a review of the related (and rather scarce) literature [5, 6, 7] shows that no reliable evaluation of their performance for high-energy particle detection exists. The devices studied in these works were all designed for detecting large doses of X-Rays, and not single minimum ionizing particles (MIPs), which generate a substantially weaker signal.

The aim of this work is a more in-depth study of the feasibility and the possible advantages of a Graphene- (or $\mathrm{MoS}_{2}$ ) based pixel detector. Possible factors favouring this kind of devices are the possibility to have signal pre-amplification integrated in the sensing device, a higher signal-to- 
noise ratio (compared to standard pixels), and a potentially easier fabrication process (compared to silicon-based preamplifiers integrated in the pixel).

\subsection{Proposed Devices}

A conceptual scheme of the proposed devices is shown in Fig.1. The detector is based on the usual ionization mechanisms in bulk semiconductors ( $\mathrm{Si}, \mathrm{Ge}$, etc...), but instead of collecting the resulting charges, it relies on a 2D material-based field effect transistor (FET) for the production of a signal. The idea is that the e-h pairs produced in Si by incoming MIPs produce a field-effect modulation of the current passing in the 2D channel of the FET.

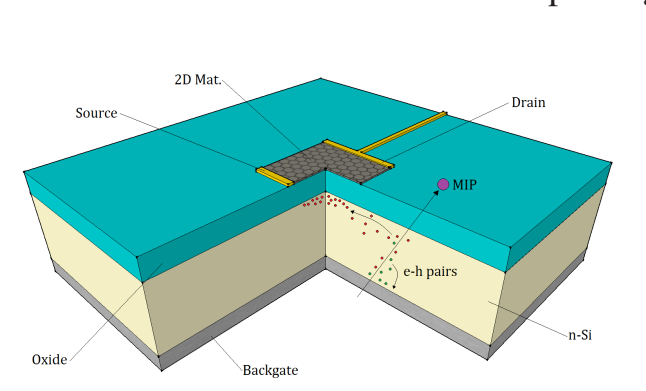

Figure 1: Principle of operation The device is composed of a high-resistivity $\mathrm{Si}$ substrate with a thin $\mathrm{SiO}_{2}$ layer on top. A FET made with a 2D material of choice is located on the oxide, and constitutes the sensing element. When a particle hits the device, part of its lost energy goes into the production of e-h pairs that drift towards the surface of the sensor thanks to an applied electric field. The field generated by the ionization charges shifts the Fermi level of the $2 \mathrm{D}$ channel, leading to a variation of its conductivity and therefore to a modulation of the output current of the transistor. Candidate materials for these devices could be Graphene or TMDs, which have interesting electronic properties and can be grown in large areas.

\section{Simulation Methods}

\subsection{D Materials Modelling}

Numerical simulations were used to assess the radiation sensitivity of the proposed devices, mainly using Synopsys SENTAURUS TCAD ${ }^{\circledR}[8]$ and NanoTCAD ViDES [9, 10] for preliminary calculations. Since no support for $2 \mathrm{D}$ materials is present in the material library provided for Sentaurus, two artificial materials were defined, with a 3D-carrier density $n_{3 D}$ such as that, being $d$ the region thickness, the product $n_{3 D} \cdot d$ gives the correct 2D-carrier density in the energy range of interest. To define the density of states (DoS), the "MultiValley" model [8] was employed, including a non-parabolicity correction to account for the linear dispersion in graphene: $\hbar^{2} k^{2} /\left(2 m^{*}\right)=E(1+\alpha E)$, where $k$ is the wave vector, $\hbar$ the reduced Planck constant, $m^{*}$ is the effective mass, $E$ the energy and $\alpha$ an appropriate fitting parameter. A fit of the analytical carrier density of Graphene and $\mathrm{MoS}_{2}$ with the expression of the MultiValley carrier densities was used to obtain the final parameters. As can be seen in Fig.2, the model gives a good fit of the actual 2D carrier density in a $0.5 \mathrm{eV}$ range around the Dirac Point (Graphene) or the bandgap edges $\left(\mathrm{MoS}_{2}\right)$, which is sufficient to cover the expected Fermi level shift range. The other physical quantities for the two materials were taken from the literature, assuming realistic average values for carrier mobilities. The full set of parameters is given in tab.1. 

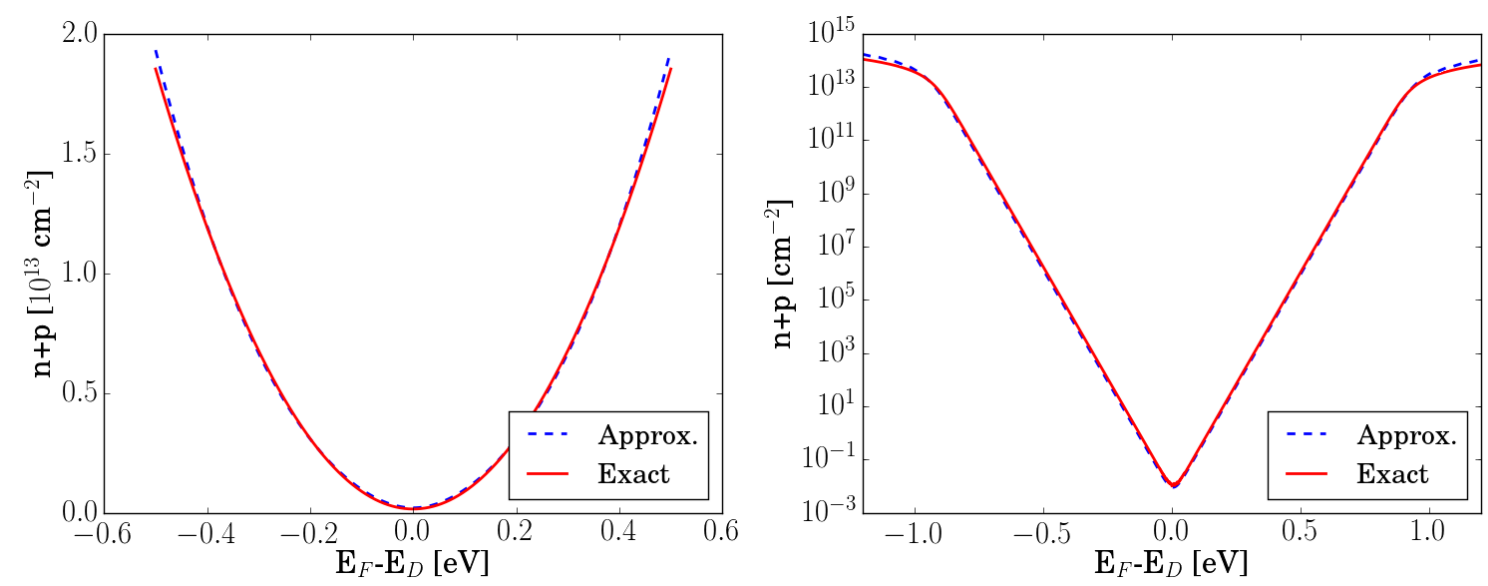

Figure 2: ML Graphene (left) and $\mathrm{MoS}_{2}$ (right) carrier densities: theoretical result (red) and approximated model (blue)

Table 1: Graphene- and $\mathrm{MoS}_{2}$-equivalent material parameters

\begin{tabular}{ccc} 
Parameter & Graphene & $\mathrm{MoS}_{2}$ \\
\hline$E_{g}$ & $10^{-4}[\mathrm{eV}]$ & $1.8[\mathrm{eV}]$ \\
$\chi$ & $4.6[\mathrm{eV}]$ & $4.26[\mathrm{eV}]$ \\
$m_{n}, m_{p}$ & $0.2024,0.2024$ & $0.54,0.44$ \\
$N_{n}, N_{p}$ & 10,10 & 1,2 \\
$\alpha$ & $1.789\left[\mathrm{eV}^{-1}\right]$ & $0.0\left[\mathrm{eV}^{-1}\right]$ \\
$d$ & $0.05[\mathrm{~nm}]$ & $0.5[\mathrm{~nm}]$
\end{tabular}

\subsection{Simulation Procedure}

The simulation calculates charge generation and transport, as well as potentials and charges in all the regions of the device. Since the newly introduced materials only have the correct carrier densities and a few other properties of the real materials, the simulation of their transport behaviour in the software would be unreliable. Therefore only the electrostatics was simulated. The potential $V_{c}$ along the Graphene/ $\mathrm{MoS}_{2}$ channel was then extracted and put inside an analytical model to calculate the resulting drain-source current. The model for the Graphene FET is based on ref.[11], and adapted to also account for $\mathrm{MoS}_{2}$ properties according to ref.[12].

To simulate a MIP losing energy in the sensor, an ultra-short optical excitation was used, with $\lambda=1.06 \mu \mathrm{m}$ and pulse intensity, beam size and duration chosen in order to produce the correct number of e-h couples $\left(\sim 2.4 \cdot 10^{4}\right.$ for $\left.300 \mu \mathrm{m}\right)$.

\section{Simulation Results}

Simulations of different structures were run considering a $50-\mu \mathrm{m}$ pitch, which is a realistic value for modern-day pixel detectors, and a standard 300- $\mu \mathrm{m}$ thickness of the Si layer. The absorber is made of $\mathrm{n}$-silicon with a doping of $10^{12} \mathrm{~cm}^{-3}$. Promising structures were simulated using both Graphene and $\mathrm{MoS}_{2}$ channels. 
Undoped substrate-devices like in [13, 7] suffered from the lack of a large depletion zone, resulting in slow response times, of the order of $20 \mu$ s due to the weak collection field (and coherent with diffusion-driven motion times in $\mathrm{Si}$ ). The relative change in current was negligible, with a modulation less than $1 \%$ for a single MIP crossing the sensor. Another problem was the need for a reset mechanism: ionization charges accumulate at the $\mathrm{Si}_{/} \mathrm{SiO}_{2}$ interface, and remain there until recombination restores the initial state, leading to a long dead time.

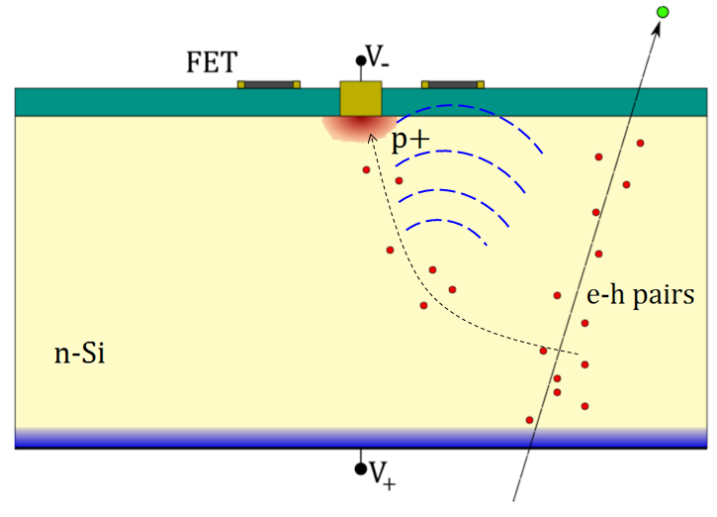

Figure 3: Schematic of the proposed pixel

Adding a p+ implant and reverse biasing the device allows full depletion, and also provides a method to carry away the charges, achieving an automatic reset. Instead of making the charges settle at the $\mathrm{Si} / \mathrm{SiO}_{2}$ interface, here the charges are carried away as soon as they reach the contacts and the response time is drastically reduced to a a peaking time around $7 \mathrm{~ns}$. A crucial parameter for the intensity of the effect turned out to be the placement of the depletion implants. The semimetallic behaviour of heavily doped $\mathrm{p}-\mathrm{Si}$ regions almost completely screens the FET from the potential generated by ionization charges. The best position for the implants was found to be beyond the FET, along the trajectory of the charges (see Fig.3). This way, apart from the area shadowed by the top contact, wherever the charges are generated inside silicon, their path before being collected passes near the G-FET/MoS 2 -FET.
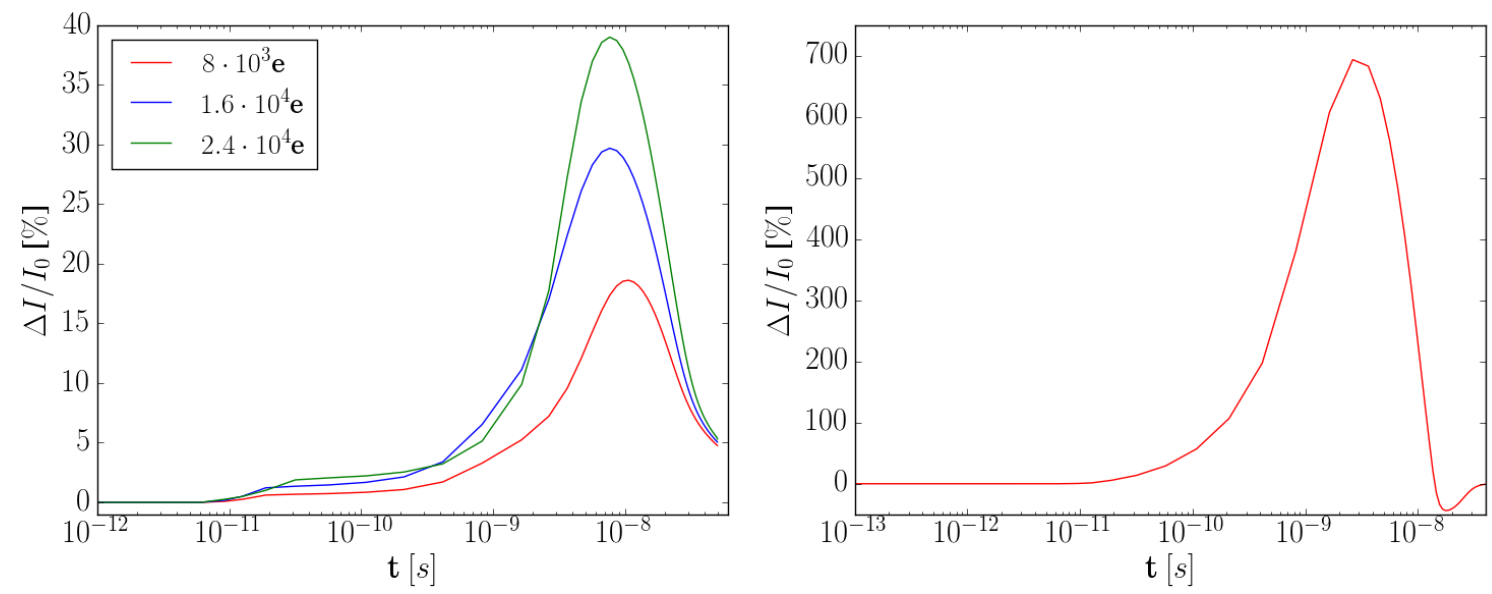

Figure 4: Current modulation for $\sim 8000, \sim 16000$ and $\sim 24000$ e-h generated couples in a pixel cell with a Graphene channel (left) or for $\sim 24000$ couples in the case of $\mathrm{MoS}_{2}$ (right).

From a simulation of a particle passing through the graphene-based device at approximately $15 \mu \mathrm{m}$ from the center, a current modulation $\Delta I / I_{0} \approx 28 \%$ was found for a full MIP (see Fig.4), for a base current $I_{0} \approx 10 \mu \mathrm{A}$ and a source to drain voltage $V_{D S}=100 \mathrm{mV}$. The current $I_{D S}$ grows when the particle hits, and the signal peaks after approximately $7 \mathrm{~ns}$, which is comparable to the peaking time of the current flowing out from the electrodes. After $\sim 60 \mathrm{~ns}$, the device has returned to its idle 
state, with the potential on the channel going back to the starting value. These timings are mostly determined by the transit time of electrons and holes in silicon, as the current flowing from the polarization electrodes has a similar time dependence. The simulation was repeated considering a $\mathrm{MoS}_{2}$ channel. While the time response is comparable, the amplitude of the modulation is much larger, reaching an almost seven-fold change (see Fig.4) for a $7.7 \mu \mathrm{A}$ base current and the same $V_{D S}=100 \mathrm{mV}$. The larger variation is due to $\mathrm{MoS}_{2}$ bandgap, which keeps current very low in the steady state.

\subsection{Pixel Array}

These devices could be arranged in a configuration similar to the one in Fig.5a to make a pixel matrix. The "unit" is a pair of cells with specular geometries, where the bias is given by a common backgate and a series of electrode/implant lines which divide the device into strips. Along each strip there is a periodic arrangement of FETs. In order to limit the number of contacts, all the source electrodes are in common, while each drain will have its own connection to the readout electronics.

A 2D simulation of a section of the device along the axis perpendicular to the strip direction was run to assess the variation in sensitivity for different hit points: as shown in Fig.5b, there is an almost $60 \%$ variation, with the minimum being right beneath the polarization implant.
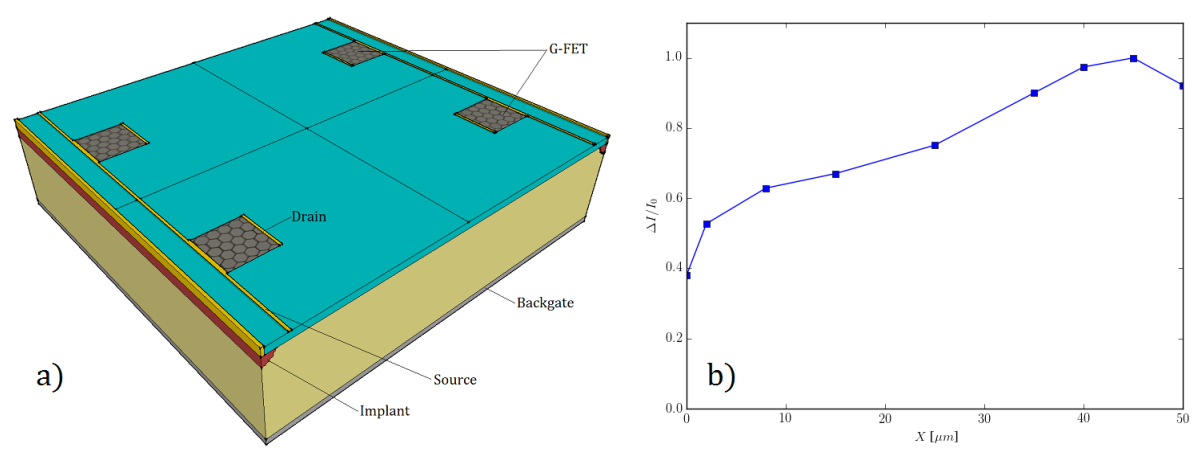

Figure 5: Schematic of a possible pixel matrix (a) and normalized pixel sensitivity $\Delta I / I_{0}$ as a function of the impact point distance from the implant line (b)

\section{Noise Figure}

A readout circuit for the simulated devices could be similar to the one shown in Fig.6. Here $C_{D}, C_{I}, C_{F}$ are the detector capacitance, the input capacitance and the feedback capacitance. A typical shaping system $\left(\mathrm{CR}-\mathrm{RC}^{2}\right)$ is considered, with a time constant $\tau$. The detector is represented as a current source $I_{0}$ emitting a delta-shaped signal $I(t)=Q_{\text {in }} \delta(t)$, where $Q_{\text {in }}$ is the total charge carried by the current pulse: $Q_{\text {in }}=\int \Delta I(t) d t$. The delta-approximation is realistic when $\tau$ is longer than the original signal duration $\Delta t_{s} \approx 60 \mathrm{~ns}$, and thus one can assume full charge collection and neglect ballistic loss. 


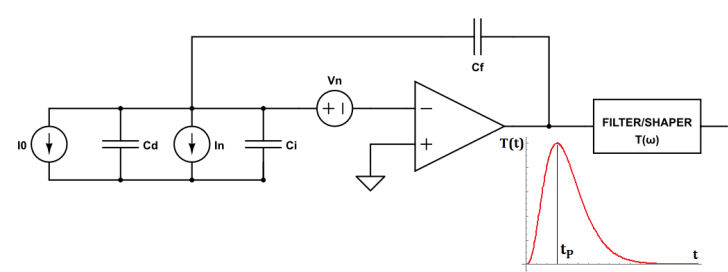

Figure 6: Readout circuit

The circuit integrates the current on the capacitor $C_{F}$, resulting in a final output $V(t)=$ $-\frac{Q}{C_{F}} \mathscr{L}^{-1}[T(s) / s]$ ( $\mathscr{L}$ is the Laplace transform). The calculation was done for the graphene-based device, since it would be the simpler to build due to more advanced production techniques. Noise is modelled with two uncorrelated sources $V_{n}$ and $I_{n}$, which include contributions from white and flicker noise coming from the detector and the preamplification circuit. The resulting SNR ratio is $\mathrm{SNR}=Q_{\text {in }} / \mathrm{ENC}$, where $Q_{\text {in }}$ is the total charge coming from the integration of the output current of the FET:

$$
Q_{\mathrm{in}}=Q_{\mathrm{FET}}=\int \Delta I d t \approx 79 \mathrm{fC}
$$

with an amplification factor of about 20 over the ionization charge $Q_{\text {ion }} \approx 3.8 \mathrm{fC}$.

This built-in amplification greatly improves detection capabilities, by increasing the effective input charge. The total ENC is mainly controlled by the parallel noise contribution, arising from the thermal noise inside the detector. This results in having an ENC minimum at $t_{P}$ values smaller than the signal duration, with $t_{P_{\mathrm{MIN}}}<20 \mathrm{~ns}$. Such an interval is too short to be used, and also would mean to discard most of the pre-amplified input charge, as well as to break the delta-approximation introduced. Thus, the system could be used at $t_{P}$ between 80 and 100 ns. For peaking times below this values one should take into account the real time profile of the input signal. From typical parameters of modern readout systems (tab.2) the calculations above give ( $t_{P}=100 \mathrm{~ns}$ ) $\mathrm{ENC} \approx 1700 \mathrm{e}^{-}$, leading to a signal-to-noise ratio

$$
\mathrm{SNR}=\frac{4.93 \cdot 10^{5} \mathrm{e}^{-}}{1700 \mathrm{e}^{-}} \approx 290
$$

As a comparison, the ATLAS Pixel detector uses $250 \mu \mathrm{m}$ thick sensors with 50x400 $\mu \mathrm{m}^{2}$ pixels with a typical ENC of $200 \mathrm{e}^{-}$, corresponding to a SNR of about 100 [2].

\begin{tabular}{cc} 
Parameter & Value \\
\hline$C_{F}$ & $150[\mathrm{fF}]$ \\
$C_{I}, C_{D}$ & $50[\mathrm{fF}]]$ \\
$A_{W}$ & $10^{-16}\left[\mathrm{~V}^{2} / \mathrm{Hz}\right]$ \\
$A_{f}$ & $10^{-11}\left[\mathrm{~V}^{2} / \mathrm{Hz}\right]$ \\
$B_{W}$ & $4 k_{B} T g_{m}\left[\mathrm{~A}^{2} / \mathrm{Hz}\right]$
\end{tabular}

Table 2: Readout circuit parameters

\section{Conclusion}

The results obtained in the simulations are promising, and suggest that further study is required. Simulated devices show a strong response to incoming MIPs, while being relatively simple in their structure and compatible with standard readout circuits. Although the presented noise estimates for the graphene-based pixels use a simplified model and may be overestimating SNR, the 
figures obtained show great potential. The next step would be to fabricate devices, and test them with exposure to a radiation source. Other interesting development could come from the use of different 2D materials (like transition metal dichalcogenides) and substrates (SiC, Diamond, CdTe or CdZnTe...), allowing to match the detector characteristics to a specific purpose, like MIP-, X-Raysor Gamma-detection.

However, some aspects still require improvement. The realization of a full pixel detector module is subordinated to technological advancements in the production process of 2D materials. Should these goals be achieved, devices such as those presented here could be attractive, since their fabrication would be less elaborate than those of monolithic pixels being investigated at the moment. A favourable aspect in this regard is that the requirements in terms of mobility and scattering are pretty low, as shown in the simulations, where values at least an order of magnitude lower than the best reported in literature for $\mu$ were used.

\section{Acknowledgments}

The authors would like to thank Prof. Valerio Re (UNIBG) for his help with the noise evaluation.

\section{References}

[1] N. Wermes. Pixel detectors for charged particles. Nuclear Instruments and Methods in Physics Research Section A: Accelerators, Spectrometers, Detectors and Associated Equipment, 604(1âĂŞ2):370 - 379, 2009. \{PSD8Proceedings\} of the 8th International Conference on Position Sensitive Detectors.

[2] Timon Heim. Status and performance of the \{ATLAS $\}$ pixel detector after 3 years of operation. Nuclear Instruments and Methods in Physics Research Section A: Accelerators, Spectrometers, Detectors and Associated Equipment, 765:227 - 231, 2014.

[3] Marco Battaglia et al. R\&d paths of pixel detectors for vertex tracking and radiation imaging. Nuclear Instruments and Methods in Physics Research Section A: Accelerators, Spectrometers, Detectors and Associated Equipment, 716:29 - 45, 2013.

[4] FHL Koppens, T Mueller, Ph Avouris, AC Ferrari, MS Vitiello, and M Polini. Photodetectors based on graphene, other two-dimensional materials and hybrid systems. Nature nanotechnology, 9(10):780-793, 2014.

[5] Michael Foxe, Gabriel Lopez, Isaac Childres, Romaneh Jalilian, Amol Patil, Caleb Roecker, John Boguski, Igor Jovanovic, and Yong P Chen. Graphene field-effect transistors on undoped semiconductor substrates for radiation detection. Nanotechnology, IEEE Transactions on, 11(3):581-587, 2012.

[6] Edward Cazalas, Biddut K Sarker, Michael E Moore, Isaac Childres, Yong P Chen, and Igor Jovanovic. Position sensitivity of graphene field effect transistors to x-rays. Applied Physics Letters, 106(22):223503, 2015.

[7] Alessandra Di Gaspare, Antonio Valletta, Guglielmo Fortunato, Rosanna Larciprete, Luigi Mariucci, Andrea Notargiacomo, and Roberto Cimino. Graphene-based field effect transistors for radiation-induced field sensing. Nuclear Instruments and Methods in Physics Research Section A: Accelerators, Spectrometers, Detectors and Associated Equipment, 2015. 
[8] Synopsys, Inc. Sentaurus Device User Guide, version j-2014.09 edition, September 2014.

[9] Gianluca Fiori and Giuseppe Iannaccone. Ultralow-voltage bilayer graphene tunnel fet. Electron Device Letters, IEEE, 30(10):1096-1098, 2009.

[10] Gianluca Fiori and Giuseppe Iannaccone. On the possibility of tunable-gap bilayer graphene fet. Electron Device Letters, IEEE, 30(3):261-264, 2009.

[11] S. A. Thiele, J. A. Schaefer, and F. Schwierz. Modeling of graphene metal-oxide-semiconductor field-effect transistors with gapless large-area graphene channels. Journal of Applied Physics, 107(9), 2010.

[12] Gianluca Fiori, BartholomÃd'us N. Szafranek, Giuseppe Iannaccone, and Daniel Neumaier. Velocity saturation in few-layer mos2 transistor. Applied Physics Letters, 103(23), 2013.

[13] Mike Foxe, Gabriel Lopez, Isaac Childres, Romaneh Jalilian, Caleb Roecker, John Boguski, Igor Jovanovic, and Yong P Chen. Detection of ionizing radiation using graphene field effect transistors. IEEE Transactions on Nanotechnology, 581:11, 2012. 